BONPLANDIA 20(2). 2011

ISSN: 0524-0476

\title{
ADAPTÓGENOS: PLANTAS MEDICINALES TRADICIONALES COMERCIALIZADAS COMO SUPLEMENTOS DIETÉTICOS EN LA CONURBACIÓN BUENOS AIRES-LA PLATA (ARGENTINA)
}

\author{
PATRICIA M. ARENAS ${ }^{1,2}$, INÉS CRISTINA ${ }^{1}$, JEREMÍAS P. PUENTES ${ }^{1}$, FERNANDO BUET COSTANTINO ${ }^{1}$, JULIO A. \\ HURRELL ${ }^{1,3}$, MARÍA LELIA POCHETTINO ${ }^{1,3}$
}

\begin{abstract}
Summary: Arenas, P. M., I. Cristina, J. P. Puentes, F. Buet Costantino, J. A. Hurrell \& M. L. Pochettino. 2011. Adaptogens: traditional medicinal plants commercialized as dietary supplements in the conurbation Buenos Aires-La Plata (Argentina). Bonplandia 20(2): 251264.

This paper presents the results of a study on three species, of which commercialize products considered adaptogens: Lepidium meyenii Walp. (Brassicaceae), "maca", Morinda citrifolia L. (Rubiaceae), "noni", and Petiveria alliacea L. (Phytolaccaceae), "pipi". The data come from an Urban Ethnobotany study conducted in Buenos Aires-La Plata conurbation. The traditional uses of the species, their access routes, marketing and diffusion as dietary supplements in urban pluricultural context are presented, as well as current modes of use and related knowledge which are part of local botanical knowledge. Thus, modifications in the uses originally assigned as result of the expansion of the products in the context of globalization are evaluated, well as the consequent changes in traditional patterns of knowledge, beliefs and practices related to these plant resources.
\end{abstract}

Key words: Urban Ethnobotany, botanical knowledge, Lepidium meyenii, Morinda citrifolia, Petiveria alliacea.

Resumen: Arenas, P. M., I. Cristina, J. P. Puentes, F. Buet Costantino, J. A. Hurrell \& M.L. Pochettino. 2011. Adaptógenos: plantas medicinales tradicionales comercializadas como suplementos dietéticos en la conurbación Buenos Aires-La Plata (Argentina). Bonplandia 20(2): 251-264.

En el presente trabajo se presentan los resultados de un estudio sobre tres especies de las cuales se comercializan productos considerados adaptógenos: Lepidium meyenii Walp. (Brassicaceae), "maca", Morinda citrifolia L. (Rubiaceae), "noni", y Petiveria alliacea L. (Phytolaccaceae), "pipi”. Los datos provienen de estudios en Etnobotánica urbana realizados en la conurbación Buenos Aires-La Plata, se presentan los usos tradicionales de las especies, sus vías de ingreso, comercialización y difusión como suplementos dietéticos en el contexto pluricultural urbano, los modos actuales de empleo y los saberes relacionados, que forman parte del conocimiento botánico local. De este modo, se evalúan las modificaciones en los usos originalmente asignados, como consecuencia de la expansión de los productos en el marco de la globalización, y los cambios consecuentes en los patrones tradicionales de saberes, creencias y prácticas referidos a estos recursos vegetales.

Palabras clave: Etnobotánica urbana, conocimiento botánico, Lepidium meyenii, Morinda citrifolia, Petiveria alliacea.

${ }^{1}$ Laboratorio de Etnobotánica y Botánica Aplicada (LEBA), Facultad de Ciencias Naturales y Museo, Universidad Nacional de La Plata, Calle 64 nro. 3, 1900, La Plata, Buenos Aires, Argentina.

${ }^{2}$ Profesional Principal CONICET.

${ }^{3}$ Investigador CONICET.

E-mail: parenas@fcnym.unlp.edu.ar 


\section{Introducción}

La Etnobotánica es la ciencia que investiga las relaciones entre los seres humanos y las plantas, en su sentido más amplio (Hurrell, 1987; Albuquerque \& Hurrell, 2010) y se ocupa, entre otros temas, del estudio del conocimiento botánico (CB), es decir, el conjunto de saberes y creencias que tienen las personas acerca de las plantas, partes de las mismas y sus productos derivados. En general, los estudios sobre el CB apuntan al de las sociedades tradicionales, porque muchas veces el $\mathrm{CB}$ tradicional (CBT) se halla en vías de extinción, por lo cual urge su recuperación.

El CBT es característico de contextos culturales homogéneos, con larga experiencia en el ambiente; se transmite de generación en generación, de forma oral y en las prácticas compartidas $\mathrm{y}$, dado que quienes consumen, producen, la relación entre producción y consumo es directa (Balick \& Cox, 1996). E1 CBT es adaptativo, porque permite los ajustes del grupo a las circunstancias cambiantes del entorno; no es estático ni conservativo, sino dinámico e innovador: evoluciona (Castellano, 2000; Pochettino \& Lema, 2008, Hurrell $\&$ al., 2010). No obstante, otras situaciones vinculadas con el mismo tipo de procesos, como la incorporación de nuevos saberes, la transferencia de usos de unas plantas a otras, los cambios en los destinos asignados a los elementos vegetales han sido poco analizadas. Estas situaciones son frecuentes en ámbitos urbanos, contextos pluriculturales sin una extensa experiencia del grupo humano en el ambiente (en términos comparativos con los contextos culturales homogéneos), en los cuales el CB se transmite a través de los medios de comunicación. En estos contextos, la relación entre producción y consumo es indirecta: quienes consumen, no producen, y a los productos vegetales circulantes, por lo común, se los considera recursos no tradicionales (Ladio \& Molares, 2010). Sin embargo, en los ámbitos urbanos, junto al CB no tradicional (que incluye el conocimiento científico), coexisten otros saberes vinculados a las tradiciones familiares o las de distintos grupos de inmigrantes (tradiciones ligadas a sus lugares de origen). Así, el $C B$ urbano (CBU) es un corpus complejo de conocimientos diversos (no tradicionales, ligados a tradiciones) que interactúan en el mismo marco pluricultural. El CBU es también adaptativo, orienta la elección de ciertos productos, en tanto otros se desechan. Su estudio es un tema central de la Etnobotánica urbana (Hurrell \& al., 2011b; Pochettino \& al., 2008, 2011).

Este trabajo se enmarca en una línea de investigación en Etnobotánica urbana del Laboratorio de Etnobotánica y Botánica Aplicada (LEBA), Facultad de Ciencias Naturales y Museo, Universidad Nacional de La Plata, donde se desarrollan estudios referidos al $\mathrm{CB}$ ligado a tradiciones, en especial, de grupos de inmigrantes (por ejemplo, de origen andino y asiático), en sintonía con otros estudios realizados en diversos países, incluido el nuestro (Balick \& al., 2000; Ceuterick \& al., 2008; Hurrell \& al., 2011b; Pieroni \& al., 2005; Pochettino \& al., 2011; Reiff \& al., 2003; Richeri \& al., 2010; Sandhu \& Heinrich, 2005; Yöney \& al., 2009).

\section{Objetivos}

El objetivo particular de este estudio fue evaluar los usos tradicionales y los modos actuales de empleo y comercialización de tres especies usadas en medicina tradicional, y las vías de ingreso y distribución tanto de las especies y sus productos como de los saberes relacionados. Estas especies se consideran casos ilustrativos de diferentes situaciones: Lepidium meyenii Walp. (= L. peruvianum $\mathrm{G}$. Chacón) (Brassicaceae), "maca", convertida en los últimos 30 años de cultivo relictual en Perú, a un fenómeno de ventas mundial por sus virtudes medicinales (Bianchi, 2003); Morinda citrifolia L. (Rubiaceae), "noni", con una larga tradición terapéutica en Polinesia, hoy de amplia presencia en el mercado (Dixon \& al. 1999); y Petiveria alliacea L. (Phytolaccaceae), "pipi", especie de la medicina tradicional americana, hoy promocionada en la terapia oncológica alternativa (Hurrell \& al., 2011a; Pochettino, 2004).

Los productos obtenidos a partir de estas 
especies se comercializan, por lo común, como suplementos dietéticos. Esta expresión fue introducida en 1994, en los Estados Unidos, en la Dietary Supplement Health and Education Act, DSHEA ('Ley de Suplementos Dietéticos, Salud y Educación'). Desde entonces, han irrumpido en el mercado global, y la Argentina no ha sido una excepción. En origen, se concibieron como complementos alimentarios para individuos sanos o con requerimientos especiales, como los deportistas; en la actualidad se consumen más por su relación con cuestiones estéticas, como los adelgazantes (Arenas, 2007, 2009, 2010; Pochettino \& al., 2008), o para aliviar situaciones de estrés o de cansancio, como los tónicos o revitalizantes y adaptógenos (Cristina \& Arenas, 2007, 2010).

\section{Adaptógenos}

El término adaptógeno fue acuñado en 1947 por Nicolai Lazarev para describir los efectos no esperados del dibazol (2-benzylbenzimidazol), un dilatador arterial. En estudios experimentales, se vio que esa droga aumentaba la resistencia del organismo al estrés físico, químico y biológico, y restablecía la vitalidad general. El término estrés se utiliza en su sentido clásico, como estado de desequilibrio (Panossian \& al., 1999; Rieli Mendes \& Carlini, 2007). La calidad de adaptógeno implica que ejerce un efecto regulador de la homeostasis y favorece un incremento inespecífico del sistema de defensa, a través de la estimulación de mediadores químicos, en especial, en casos de emergencia y condiciones desfavorables. Se ha indicado que la actividad antioxidante juega un importante rol en el mecanismo adaptogénico (Alonso, 2004).

Los productos con propiedades adaptogénicas se caracterizan por: 1. Carecer de toxicidad y provocar sólo pequeños cambios fisiológicos de las funciones vitales; 2. Poseer un efecto inespecífico, ya que estimulan mecanismos de defensa generalizados del organismo frente a cualquier tipo de noxa, sea esta física, química o biológica; 3 . Poseer un efecto normalizador (HMPC, 2007). Para
Montes Guyot (1990), además, un adaptógeno es efectivo si se usa de forma profiláctica. Estos productos constituyen una nueva clase de reguladores metabólicos, que favorecen la respuesta adaptativa del organismo. No obstante, muchas veces el término adaptógeno se utiliza de forma indiscriminada; a menudo, sin sustento científico (Panossian, 2003a,b).

\section{Área de estudio}

Este trabajo se desarrolló en el contexto de la conurbación Buenos Aires-La Plata, la mayor de la Argentina, que incluye dos aglomerados urbanos contiguos: el Gran Buenos Aires y el Gran La Plata (AABA, 2010). En términos administrativos, el Gran Buenos Aires comprende la ciudad de Buenos Aires (la Capital Federal) y 24 partidos de la provincia de Buenos Aires. Su área total es de $3.833 \mathrm{~km}^{2}$ (Gemini, 2003). Con $202 \mathrm{~km}^{2}$, la Ciudad de Buenos Aires tiene una población de 2.891.082 habitantes; los 24 partidos bonaerenses suman 9.910.282 habitantes (INDEC, 2011). En población, es el mayor aglomerado urbano del país, el segundo de Sudamérica, el tercero de Latinoamérica, el quinto de América, y el décimo séptimo del mundo (Forstall \& al., 2004).

El Gran La Plata se constituye en torno a la ciudad de La Plata, capital de la provincia de Buenos Aires, situada a $58 \mathrm{~km}$ de la Capital Federal. Está integrado por los partidos de La Plata, Berisso y Ensenada, su superficie total es de $1162 \mathrm{~km}^{2}$ y la población total asciende a 793.365 habitantes (INDEC, 2011).

\section{Materiales y Métodos}

El relevamiento del CB sobre las plantas y sus productos derivados considerados adaptógenos se realizó según la metodología etnobotánica habitual: técnicas de observación cuali y cuantitativas, observación participante, entrevistas abiertas y semiestructuradas (Albuquerque \& Lucena, 2004; Alexíades \& Sheldon, 1996; Martin, 1995). Estas fueron realizadas a informantes calificados, personas que expenden los productos, conocen sus 
propiedades, instruyen a los consumidores sobre sus usos y recomiendan su empleo; también, a informantes no calificados o legos: los consumidores (Pochettino \& al., 2008). Además, se revisó bibliografía pertinente sobre las especies y sus productos derivados; para algunos de los cuales se evaluó, asimismo, la información complementaria de etiquetas, catálogos y prospectos que, para el público en general, ayudan a orientar la selección de los productos a consumir.

Durante el trabajo de campo se obtuvieron muestras de distinta naturaleza, que fueron analizadas y depositadas en el Laboratorio de Etnobotánica y Botánica Aplicada (LEBA). Se relevaron 20 sitios de expendio, considerados relevantes por la diversidad de productos ofrecidos $y$, en consecuencia, resultan ampliamente visitados. En La Plata se relevaron 10 dietéticas y herboristerías; en City Bell, 1 dietética; en San Isidro, 2 dietéticas y herboristerías; en la Capital Federal, 3 dietéticas (barrios de Belgrano y Recoleta), 2 locales y 2 puestos callejeros en el mercado boliviano del barrio de Liniers. En total, se entrevistaron 40 informantes, de ambos sexos y distintos grupos etarios.

\section{Resultados y Discusión}

En la Tabla 1 se presentan los datos relevados para las tres especies consideradas, las muestras que constituyen el material de referencia depositado en el LEBA (se indica el leg. y número de muestra), los productos $\mathrm{y}$ sus formas de presentación; además, a los fines comparativos, las propiedades actualmente difundidas en el circuito comercial, las reconocidas en diversos estudios, y los principales usos tradicionales.

\section{Lepidium meyenii Walp.}

La "maca", "maca peruana", "maino" o, en quechua, "ayak chichira", "ayak willku", es una hierba perenne (bienal o anual en cultivo) con hojas basales arrosetadas y órgano subterráneo engrosado, reservante, originado en el hipocótile y la raíz (llamado comúnmente "raíz"), nativa de la zona andina de Perú, Bolivia y el Noroeste argentino (Al-Shehbaz, 2010; León, 1964; Marín-Bravo, 2003; Ochoa \& Ugent, 2001). Sus "raíces" son conocidas y empleadas desde los tiempos precolombinos por sus propiedades medicinales y su valor alimentario. En Perú, se ha cultivado entre los 3700 y $4400 \mathrm{~m}$ de altitud. Hasta 1980, se hallaba limitada a algunos enclaves de los Andes; pero, a raíz de su difusión mundial, se realizaron intentos de cultivo en Ecuador y Bolivia, en muchos casos, a partir de material reproductivo obtenido de forma ilegal (Hermann \& Bennet, 2009).

Composición química. La maca es rica en hidratos de carbono y fibras dietéticas; minerales: hierro, cobre, zinc, potasio; aminoácidos esenciales: serina, arginina, fenilalanina, valina, isoleucina, lisina; ácidos grasos: linoleico, linolénico, palmítico y oleico; también, ácidos poliinsaturados y sus amidas, macaenas y macamidas; altas concentraciones de glucosinolatos aromáticos y sus derivados, los isotiocianatos, responsables del aroma pungente de la maca cruda (Bianchi, 2003; Li $\&$ al., 2001). Se ha estudiado el efecto de los glucosinolatos en la reducción del riesgo de cáncer de próstata en animales (Gonzales \& al., 2005, 2009).

Usos tradicionales. Se tienen evidencias arqueológicas de su domesticación en Perú hacia 1600 a.C. Su valor para los antiguos peruanos se refleja en el hecho de que se utilizaba para pagar los impuestos a los administradores españoles. No obstante, su importancia residía en sus "raíces" comestibles, a modo de hortaliza, frescas o secas (conservan varios años sus propiedades); se consumen hervidas, para obtención de harina, elaboración de dulces, jugos y bebidas. En medicina tradicional se ha considerado energizante, revitalizador y estimulante de la fertilidad humana y animal (Anglese, 2007; Castro de León, 1990; Gonzales \& al., 2009; Johns, 1981; León, 1964; Marín-Bravo, 2003; Ruiz-Luna \& al., 2005; Valentova \& Ulrichova, 2003).

Ingreso al circuito comercial. En la última década del siglo $\mathrm{xx}$, el cultivo de la maca se expandió, primero, dentro de Perú, y luego fuera del país: se fomentó la exportación de distintos productos, según la demanda 
creciente del mercado (Gonzales \& al., 2009; Hermann \& Bennett, 2009). En esos años, los inmigrantes peruanos en Buenos Aires, promocionaron su valor, pero aún era difícil conseguir los productos en el país. A fines de la década de 1990 se registró una expansión mundial del consumo de esta especie y se comenzó a producir diversos suplementos dietéticos; inclusive, se utiliza la harina en la elaboración de alimentos.

La principal vía de ingreso a la Argentina de los productos elaborados con maca ha sido a través de los inmigrantes bolivianos (los productos peruanos se expandieron antes en Bolivia). Su presencia se registró primero en Jujuy, originada en el comercio fronterizo "hormiga" (Benedetti \& Salizzi, 2011). Hacia 2005, se la registró en mercados de origen boliviano en el área metropolitana de Buenos Aires, en correlación con el aumento de la inmigración boliviana en la conurbación (Cerrutti, 2009; Sassone, 2009). En el mercado boliviano del barrio de Liniers ingresan distintos productos, en forma directa desde Bolivia, por vía terrestre, hasta la terminal de ómnibus de ese barrio. Esos productos satisfacían la demanda de las comunidades boliviana y peruana $\mathrm{y}$, en poco tiempo, los productos restringidos a los inmigrantes, ingresaron al circuito comercial general, y comenzaron a venderse en las dietéticas, difundidos por los medios de comunicación (Pochettino \& al., 2011).

En la actualidad, la maca se adquiere en forma de harina o polvo, cápsulas o comprimidos, tanto en mercados tradicionales, como el de Liniers, como en diversas dietéticas de la conurbación. Los productos se promocionan por sus muy diversas "propiedades" (Tabla 1 ), en especial, como remedio vigorizante, afrodisíaco y estimulante sexual (en algunas propagandas se la denomina "viagra vegetal"). No obstante su extensa difusión en los medios, la comercialización de productos a base de maca no está autorizada por el ANMAT (2011).

Material adicional de referencia. PERÚ. Lima: Lima, Mercado de la Parada, 11-X2007, raíces, Bertone 1 (LEBA). BOLIVIA. Potosí: Potosí, mercado tradicional, 2008, raíces, Rodríguez 1 (LEBA).

\section{Morinda citrifolia $\mathbf{L}$.}

El "noni" (Hawai), "nhau" (Sudeste asiático), "nonu" (Samoa, Tonga), es un arbusto o arbolito perennifolio, con hojas elípticas, flores infundibuliformes, blancas, en cabezuelas; fruto múltiple (formado a partir de la cabezuela floral), carnoso, ovoide, amarillo en su madurez, de gusto y olor desagradable; frutos parciales drupáceos, semillas trígonas, con sacos aéreos que les permiten flotar, lo cual explicaría su dispersión hidrocora en el Pacífico. Es nativa del sur y sudeste de Asia, Filipinas, Nueva Guinea, norte de Australia e islas de la Polinesia. Ha sido introducida en cultivo en otras zonas de Oceanía (en especial, en Hawai), y en América tropical; a menudo se naturaliza (McClatchey, 2002; Ollier, 2006; Tao \& Taylor, 2011; Wang \& al., 2002).

Composición química. Contiene fenoles (damnacantal, escopoletina), ácidos orgánicos (caproico, caprílico), vitaminas $\mathrm{A}, \mathrm{C}, \mathrm{E}$, B1, B2, B6, B12, aminoácidos (como el ácido aspártico), minerales (hierro, fósforo, magnesio, sodio, calcio, cinc, cobre), xeronina, proxeronina, ácido ursólico, alcaloides, flavonoides, oligosacáridos, noradrenalina, serotonina, enzimas y otros compuestos $(\mathrm{Bu}$ \& al., 2011; Potterat \& Hamburger, 2007; Rodríguez Rivas \& al., 2005).

Usos tradicionales. Ha sido utilizada por los nativos de la Polinesia para ceremonias rituales y por su valor terapéutico; y ha tenido un rol importante en el arte de la curación ('huna') que realizaban los curanderos ('kahunas'), práctica declarada ilegal por los misioneros cristianos en Hawai a mediados del siglo XIX, conservada hasta nuestros días por tradición. Se utilizan las raíces, corteza, flores $\mathrm{y}$, en especial, el fruto, para tratar diversos problemas, como parásitos intestinales, fiebres, inflamaciones e infecciones de la piel (Abbott, 1992; Dixon \& al., 1999, McClatchey, 2002; Morton, 1992). En el Sudeste asiático se utiliza para combatir dolencias de garganta, boca y encías. En Malasia, para problemas urinarios, la diabetes y la gripe. En Filipinas, es reconocida como vermífugo. En el Caribe, es conocida como "árbol anti-dolor" y se utiliza en casos de esguinces, fracturas y fiebres. Entre los aspectos etnomédicos más 
significativos relacionados a la curación, se destaca el hecho de la estimulación que produce en el sistema inmunitario, que provoca un retraso del crecimiento de tumores como producto de la regeneración de células y el incremento de sus funciones, debido a la sinergia de los metabolitos (Bu \& al., 2011; González Lavaut \& González Lavaut, 2003; Morton, 1992; Palu \& al., 2008).

Ingreso al circuito comercial. Esta especie se introdujo desde su área de origen hacia Hawai y, luego, hacia América. Los primeros trabajos etnobotánicos que se refieren a sus usos en contextos pluriculturales datan de fines del siglo xx (Dixon \& al., 1999). En América tuvo una considerable expansión, primero en Perú, luego en Bolivia: en 2003 se registró su venta en mercados tradicionales de La Paz. En la Argentina, la presencia de productos derivados del noni (en especial, en polvo) data de 2005, en San Salvador de Jujuy, en ferias callejeras donde se ofrecía en forma de suplemento dietético. Estos productos se elaboraban en Perú e ingresan a la Argentina a través de Bolivia. A la conurbación Buenos Aires-La Plata llegaron por igual vía que la maca, y se expende en polvo, a veces llamado "harina de noni”, cápsulas, líquido, en pomada y colirio. Esos mismos productos se hallaban también en dietéticas, pero su venta fue prohibida en 2008 por el ANMAT (2011). Por este motivo, en muchas dietéticas se sugiere reemplazarlo con maca. No obstante, algunos productos se promocionan por distintos medios gráficos $\mathrm{y}$ radiales, y en Internet, como remedio "contra todos los males" y "alimento natural que provee todas las vitaminas y minerales que estimulan la autocuración".

Dado que el fruto maduro del noni tiene sabor y aroma desagradables, se desarrolló un proceso para deshidratarlo y obtener un polvo inoloro e insípido, que puede ser encapsulado. La recolección de los frutos se realiza de forma manual, se depositan en envases plásticos y se someten a un proceso de desinfección. Se elabora el extracto, se depura y se licúa con piña y miel. Una vez rectificado el $\mathrm{pH}$, se envasa y etiqueta. Las principales formas de presentación son como jugo (líquido) y extracto seco (en polvo o cápsulas); este se obtiene secando el fruto al sol o por liofilización.
Las propiedades más difundidas figuran en la Tabla 1. Si bien para algunas de sus propiedades se han realizado diversos estudios, otras parecen responder sólo a creencias populares; y otros efectos se encuentran aún en discusión en distintos círculos académicos. A pesar de que contiene compuestos con potencial actividad sobre el sistema nervioso, sus efectos neuro-psico-farmacológicos han sido poco estudiados.

Material adicional de referencia. CUBA. La Habana: La Habana, 2009, hojas, Arenas NH 1 (LEBA); La Habana, 2009, fruto, Arenas NF 2 (LEBA).

\section{Petiveria alliacea $\mathrm{L}$.}

El "pipi”, "anamú", "calauchín” o "mapurite" es una hierba perenne o subarbustiva, con hojas elípticas, flores verdosas, en espigas angostas, fruto aquenio comprimido, ápice cuneado con dos lóbulos provistos de uncínulos deflexos. Es nativa de América, desde el sur de los Estados Unidos hasta la Argentina, donde crece en las provincias del norte y noreste, hasta Entre Ríos. Se cultiva como ornamental y medicinal en áreas tropicales. Es muy aromática, y confiere mal gusto a la leche y la carne del ganado que la come (Guaglianone, 1987; Schmelzer \& GuribFakim, 2008).

Composición química. Las hojas contienen alantoína, nitrato de potasio, alcohol lignocerílico, ácido linoleico, ácido oleico, ácido palmítico, esteroides, terpenoides, saponinas. También presentan un principio activo hipoglucemiante y se ha observado que el extracto disminuye en un $60 \%$ los niveles de glucosa en sangre después de su administración oral en ratones. Las raíces y tallos contienen derivados sulfurados y bencénicos, triterpenos; la raíz, cumarinas, nitrato de potasio, ácido benzoico, beta-sitosterol, petiverina (principio amargo), alantoína y otros compuestos (Alonso \& Desmarchelier, 2005; Germosén Robineau, 2005).

Usos tradicionales. Se utilizan sus hojas y raíces. En el Noreste argentino, la corteza de la raíz, se emplea en distintas preparaciones, para tratar la fiebre, resfríos, gripe, tos y afecciones respiratorias. Con estos fines se consume en infusión o se elabora un ungüento (con 

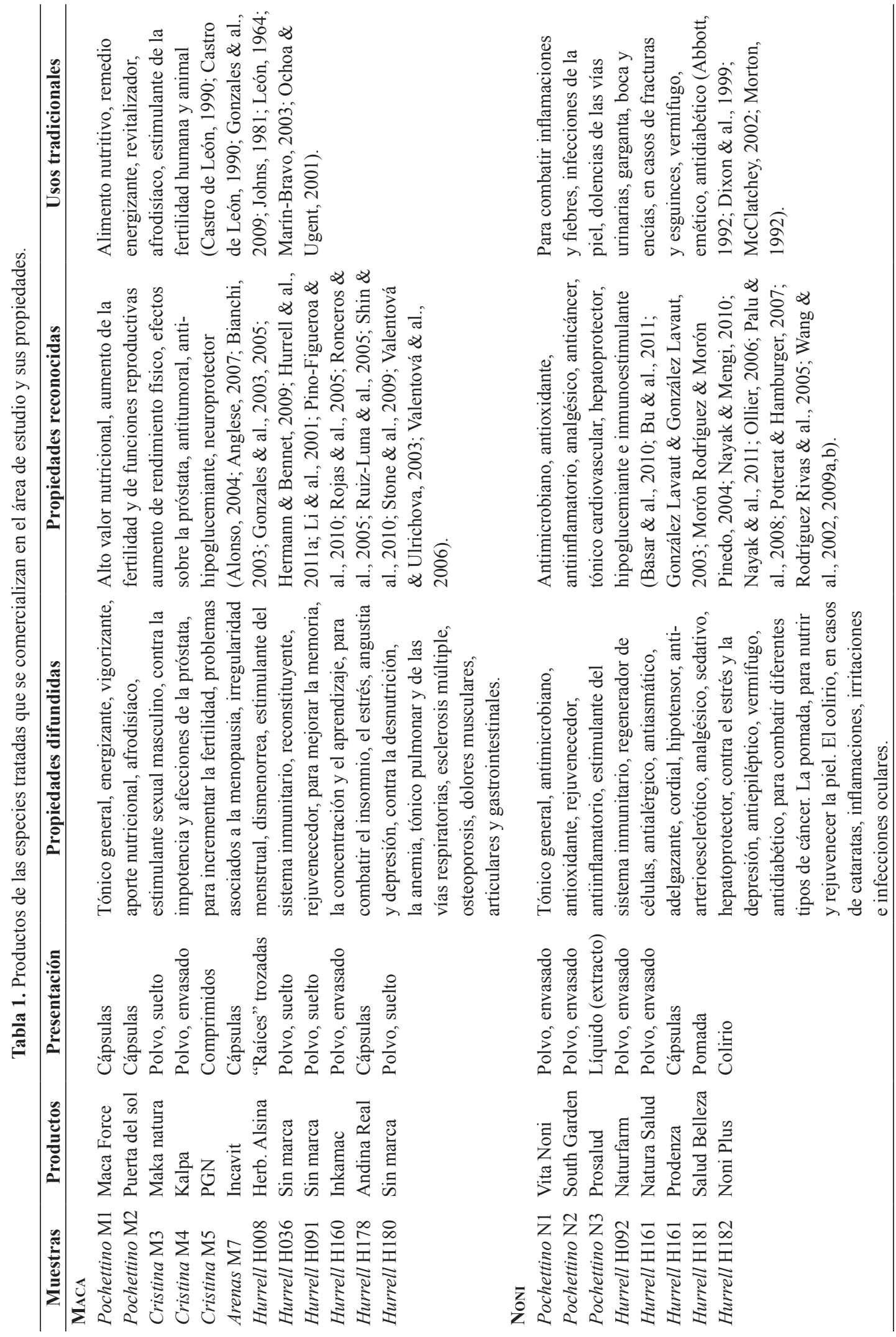

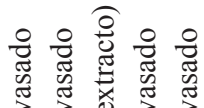

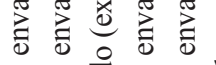

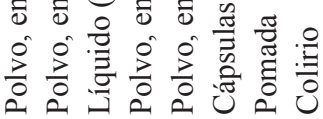

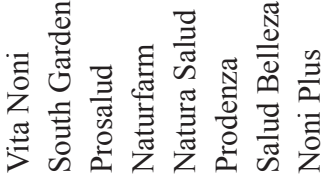

$\bar{z} \tilde{z} \tilde{Z} \widetilde{\sigma} \vec{\sigma} \bar{\infty}$

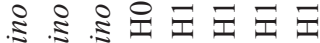

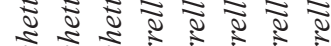

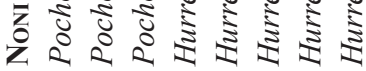


BONPLANDIA 20(2). 2011

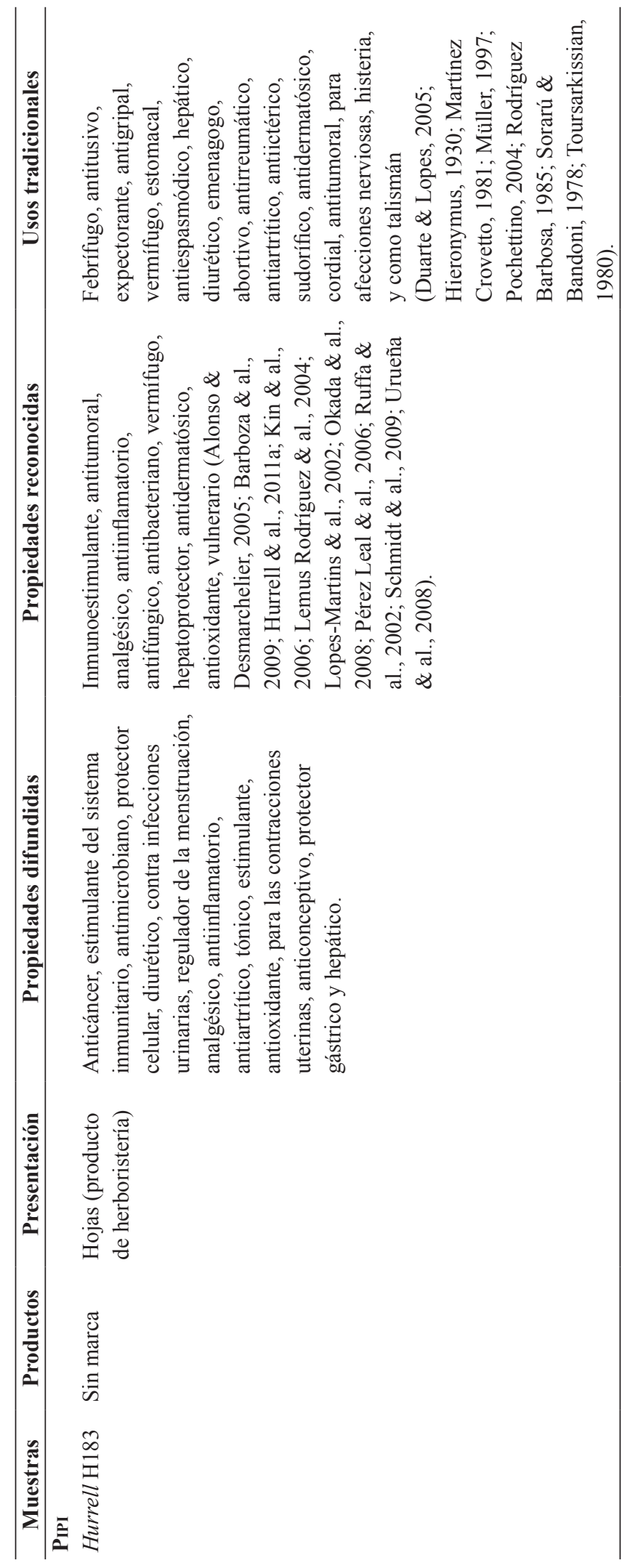


grasa de distintos animales) que se aplica en pecho y espalda. También se utiliza en casos de dolor de estómago y como vermífugo, y existen referencias de su aplicación para el dolor de oídos, como diurético, antiartrítico, antiespasmódico, abortivo, emenagogo, cordial, para problemas nerviosos, histeria, afecciones hepáticas e ictericia, nódulos mamarios (en compresas) y contra la tuberculosis (Alonso \& Desmarchelier, 2005; Barboza \& al., 2009; Hieronymus, 1930; Hurrell \& al., 2011a; Lemus Rodríguez \& al., 2004; Müller, 1997; Pochettino, 2004; Rodríguez Barbosa, 1985; Sorarú \& Bandoni, 1978; Toursarkissian, 1980). En Corrientes, la decocción de las hojas se aplica en baños en casos de espasmos y, en lavados, como antidermatósico; se prepara un decoctado antirreumático junto con hojas de naranjo agrio, aplicado en baños y fricciones. Además, se le atribuyen propiedades mágicas, como "payé" (talismán), que preserva a los habitantes de la casa contra las hechicerías (Martínez Crovetto, 1981).

Ingreso al circuito comercial. Esta especie ha integrado las farmacopeas locales de distintas zonas de la Argentina, como "pipi" en el Noreste argentino, o como "calauchin" en el Noroeste, con usos diversos. Sin embargo, en los últimos 10 años, se promociona bajo nombres foráneos, como "anamú" o "mapurite", por sus propiedades inmunoestimulante y antitumoral. Para esta última, los estudios se basaron inicialmente en el llamado "caso Managua", ocurrido en Nicaragua en 1960: centenares de vacas con leucemia fueron abandonadas a su suerte en un descampado; pasado un tiempo, los campesinos observaron que la mayoría de las vacas continuaban vivas y no presentaban señales de la enfermedad. Los animales habían consumido Petiveria alliacea, abundante en los campos (en situaciones normales se evitaba su consumo, porque el aroma y el sabor se trasmiten a la leche). Este hecho motivó investigaciones sobre su potencial en terapias oncológicas, y luego le valió reputación mundial como planta anticáncer.

En el área metropolitana de Buenos Aires, la presencia de productos es esporádica (bajo el nombre de "pipi"), y figura en algunos catálogos (como "calauchin”). Su difusión se realiza por vía oral, por sus aplicaciones en procesos inflamatorios, como la artritis, y para combatir algunos tipos de cáncer. También, se promociona en Internet, y se vende a través de esa vía, como tratamiento alternativo para el cáncer, por lo común, bajo la forma de extracto acuoso. Si bien su principal interés actual reside en su actividad antitumoral, se han estudiado asimismo otros efectos terapéuticos de interés (Tabla 1).

Material adicional de referencia. CUBA. La Habana: La Habana, 2009, hojas, Arenas PH 1 (LEBA).

\section{Conclusiones}

La denominada globalización comprende un conjunto de fenómenos diversos, políticos, económicos, culturales, que trascienden a las naciones y a los pueblos. El mundo está conformado por un conjunto de pueblos que interactúan entre sí, sobre la base de una vertiginosa aceleración de los procesos tecnológicos, en especial, la comunicación. En este marco, también se modifican los patrones tradicionales de conocimientos, creencias y prácticas referidos a los recursos naturales y su aprovechamiento.

La globalización posibilita la expansión del conocimiento a través de las diversas vías de comunicación y fomenta, sobre todo en los ámbitos urbanos, el aumento de la diversidad biocultural, donde juegan un rol fundamental los mercados y centros de expendio de productos de origen vegetal, que se constituyen en reservorios de aquella diversidad, no sólo de productos, sino de su CB asociado. En este sentido, la Etnobotánica urbana revela la complejidad del CBU, compuesto por elementos no tradicionales y otros ligados a tradiciones locales familiares, o vinculadas a segmentos de inmigrantes. Las plantas y sus productos, así como el CB subyacente, se trasladan de sus lugares de origen a nuevos contextos urbanos $\mathrm{y}$, en ese pasaje, a menudo se modifican sus usos tradicionales o se revalorizan distintos usos originales dentro del nuevo contexto urbano, como es el caso de Lepidium meyenii, utilizada desde tiempos prehispánicos con diversos 
fines, en la actualidad, revalorizada a través de su empleo como suplemento dietético. La difusión del CBT de algunas especies vegetales llega al contexto de las conurbaciones, donde adquiere un nuevo significado, como CBU ligado a tradiciones de origen, con mayor o menor expansión dentro de la población urbana local. Así, Morinda citrifolia adquiere un nuevo significado al revalorizarse como agente rejuvenecedor o adelgazante, dentro del circuito comercial, y a Petiveria alliacea se la revaloriza como planta anticáncer, más allá de sus otras y diversas utilidades terapéuticas tradicionales.

Esta modificación de algunos usos originalmente asignados dentro de los ámbitos urbanos responde, en gran medida, al rol fundamental de los medios de comunicación masiva, que instalan en la opinión pública productos vegetales ancestrales (pero novedosos para el nuevo contexto urbano). El rol de las comunidades de origen continúa siendo central en la provisión de materias primas y de muchos productos elaborados a partir de aquellas, mientras los mercados urbanos donde se comercializan (como el mercado boliviano de Liniers) se constituyen en una etapa intermedia entre las comunidades de origen y el circuito comercial globalizado. En este marco, es destacable el rol que cumplen las personas, o bien como fuente de los saberes particulares que poseen y difunden, o como elementos innovadores, a través de la adopción de nuevos recursos y utilidades.

Lewis \& Elvin-Lewis (2003) acuñaron la sigla PAT: panacea, adaptógeno y tónico. Una panacea es un remedio universal, que lo cura todo; los adaptógenos se refieren a las situaciones de estrés; y los tónicos mejoran el estado general. Según los autores, esta trilogía representa conceptos solapados, no siempre fáciles de distinguir; por ejemplo, las especies aquí tratadas, empleadas como adaptógenos en el circuito comercial global, localmente pueden considerarse panaceas. Los estudios de Etnobotánica urbana referidos a este tipo de plantas, resultan relevantes para la instalación de nuevos productos en la opinión pública y para aportar conocimientos que permitan su circulación en condiciones óptimas de calidad y de seguridad.

\section{Agradecimientos}

Los autores agradecen al Prof. E. A. Ulibarri, del Instituto de Botánica Darwinion, por su valiosa colaboración en los relevamientos de campo, así como a todos los informantes entrevistados. Este trabajo fue realizado con aportes financieros de la Universidad Nacional de La Plata y del Consejo Nacional de Investigaciones Científicas y Técnicas de la República Argentina.

\section{Bibliografía}

AABA. 2010. Atlas Ambiental de Buenos Aires. Disponible: <http://www.atlasdebuenosaires.gov. ar> [Consultado: 15-VII-2011].

ABBOTT, I. A. 1992. La‘au Hawai‘i: Traditional Hawaiian Uses of Plants. Bishop Museum Press, Honolulu. 163 pp.

AL-SHEHBAZ, I. 2010. A synopsis of the South American Lepidium (Brassicaceae). Darwiniana 48 (2): 141-167.

ALBUQUERQUE, U. P. \& J. A. HURRELL. 2010. Ethnobotany: one concept and many interpretations. In U. P. Albuquerque \& N. Hanazaki (eds.), Recent developments and case studies in Ethnobotany, pp. 87-99. SBEE-NUPEEA, Recife.

ALBUQUERQUE, U. P. \& R. F. LUCENA. 2004. Métodos e técnicas na pesquisa etnobotânica. Livro Rápido/NUPEEA, Recife. 190 pp.

ALEXÍADES, M. N. \& J. W. SHELDON. 1996. Selected guidelines for ethnobotanical research: a field manual. The New York Botanical Garden, New York. 306 pp.

ALONSO, J. 2004. Tratado de fitofármacos y nutracéuticos. Corpus, Rosario. 1350 pp.

\& C. DESMARCHELIER. 2005. Plantas medicinales autóctonas de la Argentina. Edit. Lola, Buenos Aires. 663 pp.

ANGLESE, N. 2007. Estudio de Lepidium meyenii Walp. (Maca) en suplementos dietéticos. XVI Congr. Italo-Latinoam. Etnomedicina SILAE, p. 146. La Plata.

ANMAT, 2011. Administración Nacional de Medicamentos, Alimentos y Tecnología Médica. Disponible: <http://www.anmat.gov.ar > [Consultado: 15-VII-2011].

ARENAS, P. M. 2007. Suplementos dietéticos: estudio etnobotánico en zonas urbanas. Kurtziana 33 (1): 193-202.

. 2009. Algas empleadas en la elaboración de suplementos dietéticos: abordaje etnobotánico en algunas áreas urbanas de Argentina. En P. M. 
Arenas (ed.). Etnoficología aplicada: estudio de casos en relación a la salud y la alimentación en ambientes rurales y urbanos, pp. 73-187. CYTEDRISAPRET, San Salvador de Jujuy.

- 2010. Consumo de algas en la provincia de Buenos Aires, Argentina: ¿tradición o nuevo uso de los recursos? En M. L. Pochettino, A. H. Ladio \& P. M. Arenas (eds.), Tradiciones y transformaciones en Etnobotánica, pp. 308-312. CYTED-RISAPRET, San Salvador de Jujuy.

BALICK, M. J. \& P. A. COX. 1996. Plants, people and culture. The science of Ethnobotany. Sci. Amer. Library, New York. 183 pp.

BALICK, M. J., F. KRONENBERG, A. OSOSKI, M. REIFF, A. FUGH-BERMAN, B. O'CONNOR, M. ROBLE, P. LOHR \& D. ATHA. 2000. Medicinal plants used by Latino healers for women's health conditions in New York City. Econ. Bot. 54: 344357.

BARbozA, G., J. CANTERO, C. NÚÑEZ, A. PACCIARONI \& L. ARIZA ESPINAR. 2009. Medicinal plants: A general review, phytochemical and ethnopharmacological screening of the native Argentine Flora. Kurtziana 34 (1-2): 7-365.

BASAR, S., K. UHLENHUT, P. HÖGGER, F. SCHÖNE \& J. WESTENDORF. 2010. Analgesic and antiinflammatory activity of Morinda citrifolia L. (noni) fruit. Phytother. Res. 24: 38-42.

BENEDETTI, A. \& E. SALIZZI. 2011. Llegar, pasar, regresar a la frontera. Aproximación al sistema de movilidad argentino-boliviano. Revista Transporte y Territorio 4: 148-179.

BIANCHI, A. 2003. Maca: Lepidium meyenii. Bol. Latinoamer. Caribe Plant. Medic. Aromát. 2 (3): 30-36.

BU, M., N. SÁNCHEZ, L. PÉREZ-SAAD \& I. SCULL. 2011. Efecto neurofarmacológico del zumo de Morinda citrifolia. Bol. Latinoamer. Caribe Plant. Medic. Aromát. 10 (2): 159-166.

CASTELLANO, M. B. 2000. Updating aboriginal traditions of knowledge. En G. Sefa Dei, B. L. Hall \& D. G. Rosenberg (eds.), Indigenous knowledge in global contexts. Multiple readings of our world, pp. 21-36. University of Toronto Press, Toronto.

CASTRO DE LEÓN, M. 1990. Un cultivo andino en extinción: el caso de la maca. Perú Indígena 12 (28): 85-94.

CERRUTTI, M. 2009. Diagnóstico de las poblaciones de inmigrantes en la Argentina. Ministerio del Interior. Dirección Nacional de Población, Buenos Aires. 68 pp.

CEUTERICK, M., I. VANDEBROEK, B. TORRYA \& A. PIERONI. 2008. Cross-cultural Adaptation in Urban Ethnobotany. The Colombian Folk Pharmacopoeia in London. J. Ethnopharmacol. 120: 342-359.

CRISTINA, I. \& P. M. ARENAS. 2007. Adaptógenos: ¿contribuyen al bienestar del hombre? XVI Congr. Italo-Latinoamer. Etnomed. SILAE, La Plata.

- 2010. Plantas de uso tradicional en Argentina de posible aplicación como adaptógenos. En M. L. Pochettino, A. H. Ladio \& P. M. Arenas (eds.), Tradiciones y transformaciones en Etnobotánica, pp. 282-285. CYTED-RISAPRET, San Salvador de Jujuy.

DIXON, A. R., H. MCMILLEN \& N. L. ETKIN.1999. The transformation of noni, a traditional Polynesian medicine (Morinda citrifolia, Rubiaceae). Econ Bot. 53: 51-68.

DUARTE, M. R. \& J. F. LOPES. 2005. Leaf and stem morphoanatomy of Petiveria alliacea. Fitoterapia 76: 599-607.

FORSTALL, R. L., R. P. GREENE \& J. B. PICK. 2004. Which are the largest? City Futures Conference, Chicago. 42 pp.

GEMINI, M. 2003. ¿Qué es el Gran Buenos Aires? Inst. Nac. Estadíst. Censos, Buenos Aires. 12 pp.

GERMOSÉN ROBINEAU, L. (ed.). 2005. Farmacopea vegetal caribeña. 2da. ed., Editorial UniversitariaUNAN, León. 486 pp.

GOnZAleS, G. F., A. CÓRDOVA, K. VEGA, A. CHUNG, A. VILLENA \& C. GÓÑEZ. 2003. Effect of Lepidium meyenii (maca), a root with aphrodisiac and fertility-enhancing properties, on serum reproductive hormone levels in adult healthy men. J. Endocrinol. 176 (1): 163-168.

GONZALES, G. F., S. MIRANDA, J. NIETO, G. FERNÁNDEZ, S. YUCRA, J. RUBIO, P. YI \& M. GASCO. 2005. Red maca (Lepidium meyenii) reduced prostate size in rats. Reprod. Biol. Endocrinol. 3: 5. Disponible: <http://www.rbej. com/content/3/1/5> [Consultado: 18-VII-2011].

GONZALES, G. F., C. GONZALES \& C. GONZALES CASTAÑEDA. 2009. Lepidium meyenii (maca): a plant from the highlands of Peru. From tradition to science. Forsch. Komplementmed. 16 (6): 373-380.

GONZÁlEZ LAVAUT, N. E. \& J. A. GONZÁLEZ LAVAUT. 2003. Morinda citrifolia L.: potencialidades para su utilización en la salud humana. Revista Cubana de Farmacia 37: 3. Disponible: <http://scielo.sld.cu/scielo.php> [Consultado: 9-VII-2011].

GUAGLiANONE, E. R. 1987. Phytolaccaceae. En A. Burkart, N.S. Troncoso \& N.M. Bacigalupo (eds.), Flora Ilustrada de Entre Ríos. Colecc. Cient. Inst. Nac. Tecnol. Agropec. 6 (3): 209-222.

HERMANN, M. \& T. BERNET. 2009. The transition of maca from neglect to market prominence: Lessons for improving use strategies and market chains of minor crops. Agricultural Biodiversity and Livelihoods Discussion Papers 1: I-VI, 1-101. Rome.

HIERONYMUS, J. 1930. Plantas diafóricas. Flora Argentina. 2da. ed. Atlántida, Buenos Aires. 422 pp. 
HMPC. 2007. Herbal Medicinal Products Committee. Disponible: <http://www.ema.europa.eu/ docs/en GB/document library/Scientific guideline/2009/09/WC500003646.pdf $>$ [Consulta: 28-VI-2011].

HURRELL, J. A. 1987. Las posibilidades de la etnobotánica y un nuevo enfoque a partir de la ecología y su propuesta cibernética. Revista Española de Antropología Americana (Madrid) 17: 235-258.

, E. A. ULIBARRI, G. DELUCCHI, M. L. PÉREZ \& N. ANGLESE. 2010. Plantas condimenticias utilizadas en la ciudad de Buenos Aires y en el Noreste de la Provincia de Buenos Aires (Argentina). En M. L. Pochettino, A. H. Ladio \& P. M. Arenas (eds.), Tradiciones y transformaciones en Etnobotánica, pp. 344-349. CYTED-RISAPRET, San Salvador de Jujuy.

HURRELL, J. A., E. A. ULIBARRI, P. M.ARENAS \& M. L. POCHETTINO. 2011a. Plantas de herboristería. Plantas medicinales que se comercializan en herboristerías de la Ciudad de Buenos Aires. Lola, Buenos Aires. 242 pp.

HURRELl, J. A., E. A. ULIBARRI, J. P. PUENTES, F. BUET COSTANTINO, P. M. ARENAS \& M. L. POCHETTINO. 2011b. Leguminosas medicinales y alimenticias utilizadas en la conurbación Buenos Aires-La Plata, Argentina. Bol. Latinoam. Caribe Plant. Med. Aromát. 10 (5): 443-455.

INDEC. 2011. Instituto Nacional de Estadística y Censos. República Argentina. Censo 2010. Disponible: $<$ http://www.censo2010.indec.gov.ar $>$ [Consultado 25-VI-2011].

JOHNS, T. A. 1981. The anu and the maca. J. Ethnobiol. 1 (2): 208-212.

KIN, S., R. KUBEC \& R. A. MUSAH. 2006. Antibacterial and antifungal activity of sulfurcontaining compounds from Petiveria alliacea. J. Ethnopharmacol. 104: 188-192.

LADIO, A. H. \& S. MOLARES. 2010. Aspectos do estudo da dinâmica do uso de produtos etnobiológicos não tradicionais. En U. P. Albuquerque, R. F. Lucena \& L. Cunha (eds.), Métodos e Técnicas na Pesquisa Etnobiológica e Etnoecológica, pp. 267- 278. NUPEEA, Recife.

LEMUS RODRÍGUEZ, Z., M. E. GARCÍA PÉREZ, A. BATISTA DUHARTE, O. DE LA GUARDIA PEÑA \& A. ALFONSO CASTILLO. 2004. La tableta de anamú: un medicamento herbario inmunoestimulante. MEDISAN (Santiago de Cuba) 8 (3): 57-64.

LEÓN, J. 1964. The maca (Lepidium meyenii), a little known food plant of Perú. Econ. Bot. 18: 122-127.

LEWIS, W. H. \& M. P. F. ELVIN-LEWIS. 2003. Medical Botany. Plants Affecting Human Health. 2nd. ed. Wiley \& Sons, Hoboken. 821 pp.

LI, G., U. AMMERMANN \& C. F. QUIROS. 2001.
Glucosinolate content in maca (Lepidium peruvianum) seeds, sprouts, mature plants and several derived commercial products. Econ. Bot. 55: 255-262.

LOPES-MARTINS, R. A., D. H. PEGORARO, R. WOISKY, S. C. PENNA \& J. A. SERTIÉ. 2002. The anti-inflammatory and analgesic effects of a crude extract of Petiveria alliacea L. (Phytolaccaceae). Phytomedicine 9 (3): 245-248.

MARÍN-BRAVO, M. 2003. Histología de la Maca, Lepidium meyenii Walpers (Brassicaceae). Rev. Perú Biol. 10 (1): 101-108.

MARTIN, G. 1995. Ethnobotany. A methods manual. Chapman \& Hall, London. 268 pp.

MARTÍNEZ CROVETTO, R. 1981. Las plantas utilizadas en medicina popular en el Noroeste de Corrientes (Argentina). Miscelánea Inst. Miguel Lillo 69: 27-28, Tucumán.

McCLATCHEY, W. 2002. From Polynesian healers to health food ftores: changing perspectives of Morinda citrifolia (Rubiaceae). Integr. Cancer Therapies 1 (2): 110-120.

MONTES GUYOT, M. 1990. Perspectivas de la fitoterapia. Acta Farm. Bonaerense 9 (2): 131-138.

MORÓN RODRÍGUEZ, F. J. \& D. MORÓN PINEDO. 2004. Mito y realidad de Morinda citrifolia L. (noni). Revista Cubana Plant. Med. 9: 3. Disponible: $<$ http://scielo.sld.cu/scielo.php $>$ [Consultado: 9-VI2011].

MORTON, J. F. 1992. The ocean-going noni, or Indian mulberry (Morinda citrifolia, Rubiaceae) and some of its "colorful" relatives. Econ. Bot. 46: 241-256.

MÜLLER, F. 1997. Drogas y medicamentos de los indios Guaraní (Mbyá, Pai, Chiripá) en las regiones orientales de la selva del Paraguay. Parodiana 10 (1-2): 197-209.

NAYAK, B. S. \& S. MENGI. 2010. Immunostimulant activity of noni (Morinda citrifolia) on $\mathrm{T}$ and B lymphocytes. Pharm. Biol. 48 (7): 724-731.

NAYAK, B. S., J. R. MARSHALL, G. ISITOR \& A. ADOGWA. 2011. Hypoglycemic and hepatoprotective activity of fermented fruit Juice of Morinda citrifolia (noni) in diabetic rats. Evid. Based Complement. Alternat. Med. 2011: 875293. Disponible: <http://www.hindawi.com/journals/ ecam/> [Consultado 25-VI-2011].

OCHOA, C. \& D. UGENT. 2001. Maca (Lepidium meyenii, Brassicaceae): a nutritious root crop of the Central Andes. Econ. Bot. 55: 344-345.

OKADA, Y., K. TANAKA, E. SATO \& H. OKAJIMA. 2008. Antioxidant activity of the new thiosulfinate derivative, S-benzyl phenylmethanethiosulfinate, from Petiveria alliacea L. Org. Biomol. Chem. 6 (6): 1097-1102.

OLLIER, C. 2006. Le noni aux risques de I'analyse. Phytoterapie 1: 31-42.

PALU, A. K., A. H. KIM, B. J. WEST, S. DENG, 
J. JENSEN \& L. WHITE. 2008. The effects of Morinda citrifolia L. (noni) on the immune system. Its molecular mechanisms of action. J. Ethnopharmacol. 115: 502-506.

PANOSSIAN, A. 2003a. Adaptogens, tonic herbs for fatigue and stress. Alternative \& Complement. Therapies 9 (6): 327-331.

2003b. Adaptogens: a historical overview and perspective. Natural Pharmacy 7 (4): 19-20.

—, G. WIKMAN \& H. WAGNER. 1999. Plant Adaptogens. Earlier and more recent aspects and concepts on their mode of action. Phytomedicine 6: 287-299.

PÉREZ LEAL, R, M. R. GARCÍA MATEOS, M. MARTÍNEZ VASQUEZ \& M. SOTO HERNÁNDEZ. 2006. Actividad citotóxica y antioxidante de Petiveria alliacea L. Revista Chapingo Ser. Horticult. 12 (1): 51-56.

PIERONI, A., H. MUENZ, M. AKBULUT, K. BAŞER \& C. DURMUŞKAHYA. 2005. Traditional phytotherapy and trans-cultural pharmacy among Turkish migrants living in Cologne, Germany. J. Ethnopharmacol. 102: 69-88.

PINO-FIGUEROA, A., D. NGUYE, T. J. MAHER. Neuroprotective effects of Lepidium meyenii (maca). Ann. N.Y. Acad. Sci. 1199: 77-85.

POCHETTINO, M. L. 2004. La predictividad de la Etnobotánica en un contexto globalizado. ¿Cuál es el futuro del "pipí" (Petiveria alliaceae)? Proc. III Internat. Symp. Ethnobot. Disciplines (CD), Canoas.

\& V. LEMA. 2008. La variable tiempo en la caracterización del conocimiento botánico tradicional. Darwiniana 46 (2): 227-239.

POCHETTINO, M. L., P. M. ARENAS, D. SÁNCHEZ \& R. CORREA. 2008. Conocimiento botánico tradicional, circulación comercial y consumo de plantas medicinales en un área urbana de Argentina. Bol. Latinoamer. Caribe Plant. Medic. Aromát. 7 (2): 141-148.

POCHETTINO, M. L., J. P. PUENTES, F. BUET COSTANTINO, P. M. ARENAS, E. A. ULIBARRI \& J. A. HURRELL. 2011. Functional Foods and Nutraceuticals in a Market of Bolivian Inmigrants in Buenos Aires (Argentina). Evidence-Based Complementary and Alternative Medicine, vol. 2012 (in press).

POTTERAT, O. \& M. HAMBURGER. 2007. Morinda citrifolia (noni) fruit. Phytochemistry, Pharmacology, Safety. Planta Med. 73 (3): 191-199.

REIFF, M., B. O'CONNOR, F. KRONENBERG, M. J. BALICK, P. LOHR, A. FUGH-BERMAN \& K. D. JOHNSON. 2003. Ethnomedicine in urban environment: Dominican healers in New York City. Human Organization 62 (1):12-26.

RICHERI, M., A. M. BEESKOW \& A. H. LADIO. 2010. Las plantas y la salud en la comunidad boliviana de
Puerto Madryn (Argentina). En M. L. Pochettino, A. H. Ladio \& P. M. Arenas (eds.), Tradiciones y Transformaciones en Etnobotánica, pp. 297-302. CYTED-RISAPRET, San Salvador de Jujuy.

RIELI MENDES, F. \& E. A. CARLINI. 2007. Brazilian plants as posible adaptogens: An ethnopharmacological survey of books edited in Brazil. J. Ethnopharmacol. 109: 493-500.

RODRÍGUEZ BARBOSA, N. 1985. Pojhá Ñaná. Recetario de plantas medicinales usadas en el Paraguay. 2da. ed. Asunción. 356 pp.

RODRÍGUEZ RIVAS, M., M. BOFFILL CÁRDENAS, G. LORENZO MONTEAGUDO, P. SÁNCHEZ FREIRE, L. LÓPEZ GUERRA REGLA \& B. VERDECÍA MACHADO. 2005. Evaluación preclínica del efecto antiinflamatorio del jugo de Morinda citrifolia L. Revista Cubana Plant. Med. 10: 3-4. Disponible: <http://www.sld.cu/ galerias/pdf/sitios/mednat/efecto_antiinflamatorio_ morinda.pdf $>$ [Consultado: 29-VI-2011].

ROJAS, N., G. RONCEROS, M. GARCÍA HJARLES, M. PEÑA, W. VIVANCO, O. CASTILLO, E. NEIRA \& A. CASTRO. 2005. Eficacia de Lepidium peruvianum "maca" en el tratamiento de la astenozoospermia y oligozoospermia, causas de la infertilidad masculina. An. Fac. Med. Lima 66 (Supl. 1): 33-34.

RONCEROS, G., W. RAMOS, F. GARMENDIA, J. ARROYO \& J. GUTIÉRREZ. 2005. Eficacia de la maca fresca (Lepidium meyenii Walp.) en el incremento del rendimiento físico de deportistas de altura. An. Fac. Med. Lima 66 (Supl. 1): 33.

RUFFA, M. J., G. FERRARO, M. L. WAGNER, M. L. CALCAGNO, R. H. CAMPOS \& L. CAVALLARO. 2002. Cytotoxic effect of Argentine medicinal plant extracts on human hepatocellular carcinoma cell line. J. Ethnopharmacol. 79: 335-339.

RUIZ-LUNA, A., S. SALAZAR, N. ASPAJO \& G. GONZALES. 2005. Lepidium meyenii (maca) increases litter size in normal adult female mice. Reprod. Biol. Endocrinol. 3: 16. Disponible: $<$ http:// www.rbej.com/content/3/1/16> [Consultado: 19VII-2011].

SANDHU, D. S. \& M. HEINRICH. 2005. The use of health foods, spices and other botanicals in the Sikh community in London. Phytother. Res. 19: 633-642.

SASSONE, S. 2009. Breve geografía histórica de la migración boliviana en la Argentina. En L. Maronese (ed.), Temas de patrimonio cultural 24: Buenos Aires Boliviana, pp. 389-402. Com. Preserv. Patrim. Hist. Cult. Ciudad Autónoma de Buenos Aires, Buenos Aires.

SCHMELZER, G.H. \& A. GURIB-FAKIM. 2008. Medicinal Plants. Plant Resources of Tropical Africa 11 (1): 1-791.

SCHMIDT, C., M. FRONZA, M. GOETTERT, F. GELLE, 
S. LUIK, E. M. FLORES, C. F. BITTENCOURT, G. D. ZANETTI, B. M. HEINZMANN, S. LAUFER \& I. MERFORT. 2009. Biological studies on Brazilian plants used in wound healing. J. Ethnopharmacol. 122: 523-532.

SHIN, B. C., M. S. LEE, E. J. YANG, H. S. LIM \& E. ERNST. 2010. Maca (Lepidium meyenii) for improving sexual function: a systematic review. BMC Complement. Altern. Med. 10: 44. Disponible: $<$ http://www.biomedcentral.com/1472-6882/10/44> [Consultado: 29-VII-2011].

SORARÚ, S. B. \& A. BANDONI. 1978. Plantas de la medicina popular Argentina. Albatros, Buenos Aires. $153 \mathrm{pp}$.

STONE, M., A. IBARRA, M. ROLLER, A. ZANGARA \& E. STEVENSON. 2009. A pilot investigation into the effect of maca supplementation on physical activity and sexual desire in sportsmen. J. Ethnopharmacol. 126: 574-6.

TAO, C. \& C.M. TAYLOR. 2011. Morinda. En Wu, Z. Y., P. H. Raven \& D. Y. Hong (eds.), Fl. of China 19: 220-229. Sci. Press, Beijing-Missouri Bot. Gard., St. Louis.

TOURSARKISSIAN, M. 1980. Plantas Medicinales de la Argentina. Hemisferio Sur, Buenos Aires. 178 pp.

URUEÑA, C., C. CIFUENTES, D. CASTAÑEDA, A. ARANGO, P. KAUR, A. ASEA \& S. FIORENTINO. 2008. Petiveria alliacea extracts uses multiple mechanisms to inhibit growth of human and mouse tumoral cells. BMC Complement. Altern. Med. 8: 60.
Disponible: <http://www.biomedcentral.com/14726882/8/60> [Consultado: 29-VII-2011].

VALENTOVÁ, K. \& J. ULRICHOVA. 2003. Smallanthus sonchifolius and Lepidium meyenii, prospective Andean crops for the prevention of chronic diseases. Biomed. Papers 147 (2): 119-130.

VALENTOVÁ, K., D. BUCKIOVÁ, V. KREN, J. PEKNICOVÁ, J. ULRICHOVÁ \& V. SIMÁNEK. 2006. The in vitro biological activity of Lepidium meyenii extracts. Cell Biol. Toxicol. 22 (2): 91-99.

WANG, M.Y, B. J. WEST, C. J. JENSEN, D. NOWICKI, C. SU, A. K. PALU \& G. ANDERSON. 2002. Morinda citrifolia (noni): A literature review and recent advances in noni research. Acta Pharmacol. Sin. 23 (12): 1127-1141.

WANG, M.Y., M. LUTFIYYA, V. WEIDENBACHERHOPER, G. ANDERSON, C. X. SU \& B. J. WEST. 2009a. Antioxidant activity of noni juice in heavy smokers. Chem. Central J. 3: 13. Disponible: $<\mathrm{http} / /$ journal.chemistrycentral.com/content/3/1/13> [Consultado: 9-VII-2011].

WANG, M.Y., L. PENG, M. LUTFIYYA, E. HENLEY, V. WEIDENBACHER-HOPER \& G. ANDERSON. 2009b. Morinda citrifolia (noni) reduces cancer risk in current smokers by decreasing aromatic DNA adducts. Nutr. Cancer 61 (5): 634-639.

YÖNEY,A., J. M. PRIETO,A. LARDOS \& M. HEINRICH. 2009. Ethnopharmacy of turkish-speaking cypriots in Greater London. Phytother. Res. 24: 731-740.

Original recibido el 15 de agosto de 2011; aceptado el 2 de diciembre de 2011. 